\title{
Generation of terahertz pulses with arbitrary elliptical polarization
}

\author{
N. Amer, W. C. Hurlbut, B. J. Norton, and Yun-Shik Lee ${ }^{a)}$ \\ Department of Physics, Oregon State University, Corvallis, Oregon 97331-6507 \\ T. B. Norris \\ Center for Ultrafast Optical Science, The University of Michigan, Ann Arbor, Michigan 48109-2099
}

(Received 12 July 2005; accepted 5 October 2005; published online 23 November 2005)

\begin{abstract}
We employ two different methods to generate controllable elliptical polarization of teraherz (THz) pulses. First, THz pulses are generated via optical rectification in nonlinear crystals using a pair of temporally separated and perpendicularly polarized optical pulses. The THz ellipticity is controlled by adjusting the relative time delay and polarization of the two optical pulses. We generate mixed polarization states of single-cycle $\mathrm{THz}$ pulses using ZnTe, and elliptically polarized multicycle $\mathrm{THz}$ pulses in periodically poled lithium niobate crystals. Second, we generate elliptically polarized $\mathrm{THz}$ pulses by making a THz "wave plate" using a combination of a wire-grid $\mathrm{THz}$ polarizer and a mirror to transform linearly polarized multicycle $\mathrm{THz}$ pulses into elliptical polarization. (C) 2005 American Institute of Physics. [DOI: 10.1063/1.2138351]
\end{abstract}

Studies of the interaction of coherent teraherz $(\mathrm{THz})$ pulses with matter, up to the present time, have generally employed linearly polarized $\mathrm{THz}$ waves. A wide range of new experiments, however, would be enabled if $\mathrm{THz}$ sources with controlled and adjustable elliptical polarization states were available. Examples for which the $\mathrm{THz}$ ellipticity is critical include the control of excitons in semiconductor nanostructures ${ }^{1}$ and of molecular rotational wave packets. ${ }^{2}$ Elliptically polarized $\mathrm{THz}$ is also potentially important to study macromolecular chiral structures such as proteins and DNA because of strong absorption in the $\mathrm{THz}$ regime due to collective vibrational modes. ${ }^{3}$ In spite of the potential importance, only a few efforts to control the polarization of $\mathrm{THz}$ pulses have been reported so far. ${ }^{4,5}$ The lack of established methods for generating elliptically polarized $\mathrm{THz}$ waves is partly because the most common and powerful sources such as photoconductive dipole antennas or optical rectification of ultrafast laser pulses in $\chi^{(2)}$ media naturally generate linearly polarized $\mathrm{THz}$ radiation; additionally, the majority of $\mathrm{THz}$ sources using femtosecond lasers produce single-cycle pulses and the ellipticity is not well defined for the wave form. On the other hand, ellipticity is well defined for multicycle pulses. In this letter, we describe the demonstration of elliptical polarization evolution in single-cycle $\mathrm{THz}$ pulses generated in ZnTe using pairs of linearly polarized and timedelayed femtosecond laser pulses. We also extend our recently developed technique for generating narrow-band multicycle $\mathrm{THz}$ pulses by optical rectification in periodically-poled lithium niobate (PPLN) crystals, $^{6-8}$ to generate elliptically polarized multicycle $\mathrm{THz}$ pulses. We also demonstrate a scheme to transform multicycle $\mathrm{THz}$ pulses from linear to elliptical polarization using a combination of a free-standing wire-grid (WG) $\mathrm{THz}$ polarizer and a high reflector.

The light source for these experiments was a $76 \mathrm{MHz}$ Ti:sapphire oscillator (Coherent inc., Mira 900F) producing $820 \mathrm{~nm}, 85 \mathrm{fs}$ pulses. The femtosecond pulses were focused to a spot of $20 \mu \mathrm{m}$ diameter in the nonlinear crystals. We detected the $\mathrm{THz}$ radiation using established electro-optic

\footnotetext{
${ }^{a)}$ Electronic mail: leeys@physics.orst.edu
}

sampling techniques: $\mathrm{THz}$ radiation was collimated with a pair of off-axis parabolic mirrors and focused into a $1 \mathrm{~mm}$ ZnTe crystal for electro-optic detection of the $\mathrm{THz}$ fields. ${ }^{9}$ The excitation beam was modulated at $1 \mathrm{kHz}$ for lock-in signal detection. The instantaneous $\mathrm{THz}$ polarization was determined by separately measuring the two linear components of $\mathrm{THz} E$-field vector.

In the two-pulse scheme, we control the relative phase of two linearly polarized $\mathrm{THz}$ pulses by adjusting the path length difference of the two optical pulses used to generate them. The ellipticity of the total $\mathrm{THz}$ field is thus a function of the relative time delay, intensity, and polarization angle of the two incident optical pump pulses. Even though the ellipticity is not well defined for broadband single-cycle $\mathrm{THz}$ pulses, we still can apply the two-pump-pulse scheme to generate broadband $\mathrm{THz}$ pulses of mixed polarization in $\mathrm{ZnTe}$ crystals. Since the crystal class of ZnTe is $\overline{4} 3 m$, the second order nonlinear susceptibility has six nonzero elements, of which only 1 is independent. Accordingly, the $\mathrm{THz}$ field is $\mathbf{E}_{\mathrm{THz}}=\gamma\left(E_{y} E_{z}, E_{z} E_{x}, E_{x} E_{y}\right)$ in the crystal coordinate where the applied optical field is $\mathbf{E}_{\text {opt }}=\left(E_{x}, E_{y}, E_{z}\right)$ and $\gamma$ is a coefficient proportional to the second order nonlinear susceptibility, $d_{14}$. We aligned the optical pump pulses to propagate in the $[1 \overline{1} 0]$ direction of the ZnTe crystal. The electric fields of the two perpendicularly polarized optical pulses can be written as

$$
\begin{aligned}
& \mathbf{E}_{1}(t)=\frac{E_{O}(t)}{2}(1,1, \sqrt{2}), \\
& \mathbf{E}_{2}(t)=\frac{E_{O}(t-\tau)}{2}(1,1,-\sqrt{2}),
\end{aligned}
$$

where $\tau$ is the relative time delay between the optical pulses. Then, the generated $\mathrm{THz}$ fields are

$$
\mathbf{E}_{1}^{\mathrm{THz}}(t)=\frac{E_{T}(t)}{\sqrt{5}}(\sqrt{2}, \sqrt{2}, 1),
$$



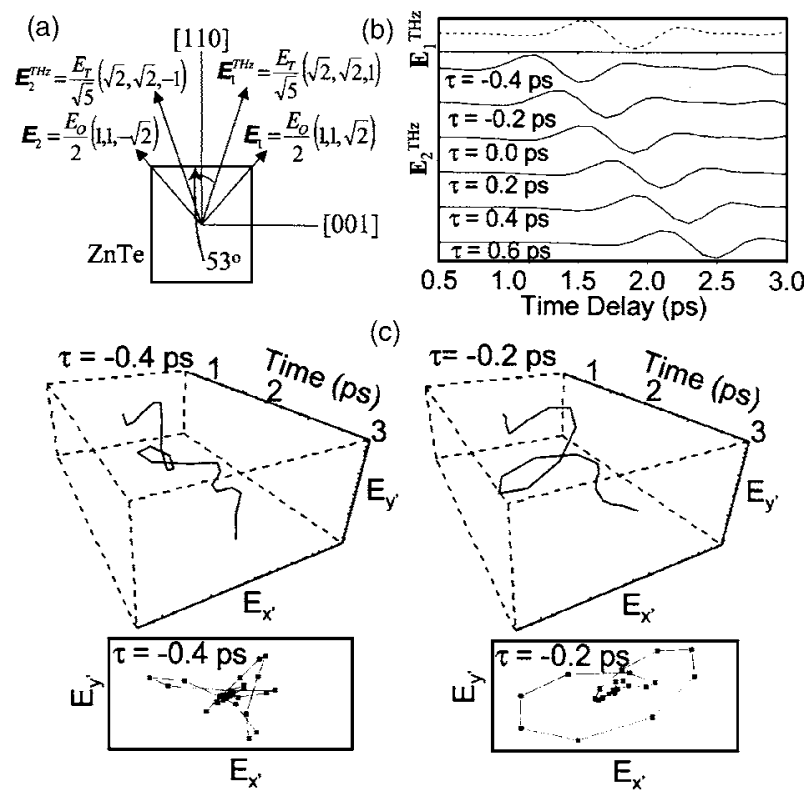

(c)
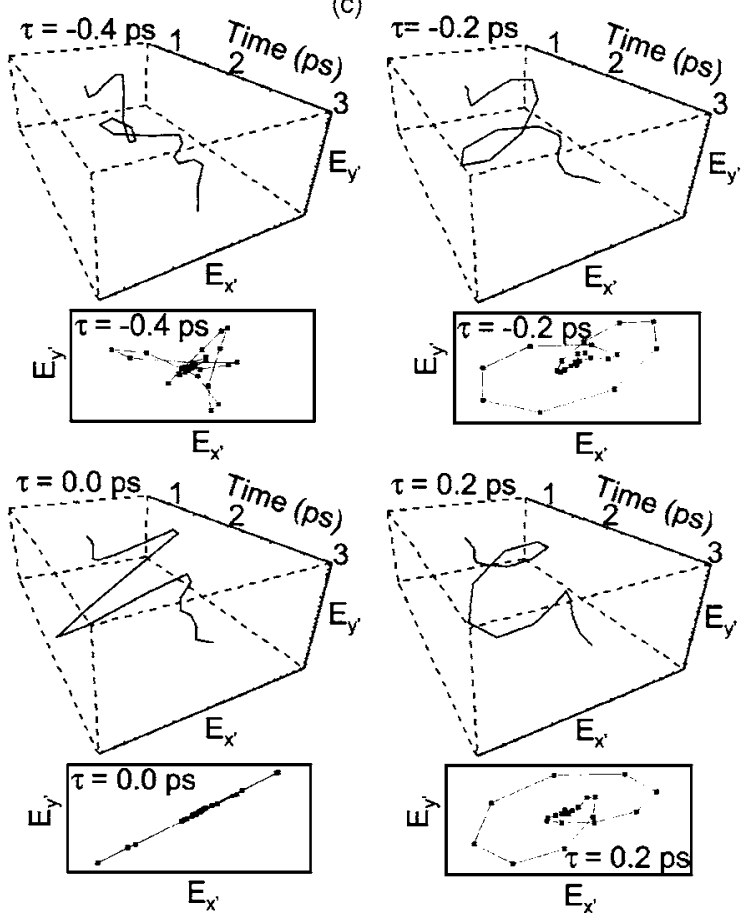

FIG. 1. Mixed polarization states of single-cycle THz pulses. (a) Optical and $\mathrm{THz}$ electric fields in ZnTe (110) plane. (b) Time-dependent wave forms of two linearly polarized $\mathrm{THz}$ pulses: the measured angle between the two $\mathrm{THz}$ fields is $51^{\circ} \pm 2^{\circ}$. (c) THz $E$-field vectors and corresponding polarization trajectories of the superposed THz fields.

$$
\mathbf{E}_{2}^{\mathrm{THz}}(t)=\frac{E_{T}(t-\tau)}{\sqrt{5}}(\sqrt{2}, \sqrt{2},-1) .
$$

The arrangement of the optical and THz fields is described in Fig. 1(a). The calculated angle between the two $\mathrm{THz}$ field vectors is $2 \tan ^{-1} \frac{1}{2}=53^{\circ}$.

The measured THz fields are shown in Fig. 1(b), revealing the temporal evolution of the two $\mathrm{THz}$ fields. The first THz pulse $\left(\mathbf{E}_{1}^{\mathrm{THz}}\right)$ is featured in the top panel, and the other $\left(\mathbf{E}_{2}^{\mathrm{THz}}\right)$ is shown below for various relative time delays $(\tau=-0.4,0.2,0.0,0.2,0.4$, and 0.6). The measured angle between the two $\mathrm{THz}$ polarizations is $51^{\circ} \pm 2^{\circ}$, close to the calculated value of $53^{\circ}$. Figure 1(c) shows the threedimensional (3D) plots of the $\mathrm{THz} E$-field vectors, and the corresponding polarization trajectories (projection of the $E$-field vector on the $x^{\prime}-y^{\prime}$ plane) of the superposed $\mathrm{THz}$ fields in the laboratory coordinate: the $\mathrm{THz}$ fields are in the $x^{\prime}-y^{\prime}$ plane and $x^{\prime}$ axis is in the direction of $1 / \sqrt{5}(\sqrt{2}, \sqrt{2}, 1)$. The time interval between the polarization trajectory data points is $0.1 \mathrm{ps}$. At zero time delay $\tau=0.0$, the superposed $\mathrm{THz}$ field is evidently linearly polarized. For other time delays, the $\mathrm{THz}$ fields have complicated polarization trajectories. When the time delays are of the same magnitude but with opposite sign, the trajectories have the same pattern, but are rotated by $180 \mathrm{deg}$ in the $x^{\prime}-y^{\prime}$ plane, e.g., $\tau=-0.2$ and
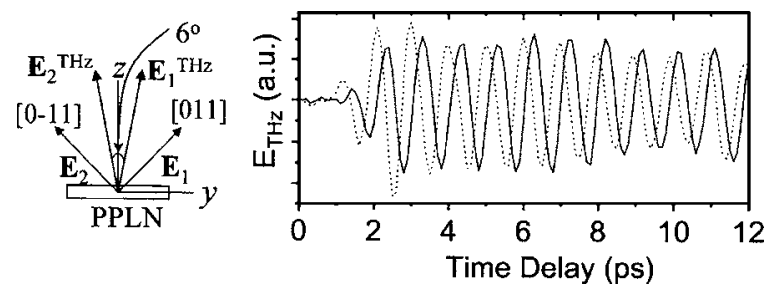

FIG. 2. Elliptically polarized narrow-band multicycle THz pulses. Two linearly and perpendicularly polarized optical pulses generate $\mathrm{THz}$ pulses $\mathbf{E}_{1}^{\mathrm{THz}}$ (dotted line) and $\mathbf{E}_{2}^{\mathrm{THz}}$ (solid line) in a PPLN crystal. The time delay $(\tau)$ between the optical pulses corresponds to $106^{\circ}$ of the phase difference $(\phi$ $=2 \pi \nu_{\mathrm{THz}} \tau, \nu_{\mathrm{THz}}=1.015 \mathrm{THz}$ ) between the $\mathrm{THz}$ pulses. The angle between the polarizations of the $\mathrm{THz}$ pulses is $6^{\circ}$.

0.2 . For $\tau= \pm 0.2$, the $\mathrm{THz} E$-field vectors show a spiral pattern. Since the mean frequency of the single-cycle THz pulse is about $1.2 \mathrm{THz}, \tau= \pm 0.2$ corresponds to the relative phase of $\pm \pi / 2$ at that frequency. The spiral pattern illustrates how the evolution of the electric field vector for a single-cycle pulse can lead to situations where the polarization ellipticity becomes ill defined.

In order to generate elliptically polarized multicycle $\mathrm{THz}$ pulses, we applied the same scheme of double-pulse excitation to optical rectification in a PPLN crystal. Figure 2 shows the polarization combination of the two linearly polarized optical and $\mathrm{THz}$ pulses, and corresponding $\mathrm{THz}$ wave forms. In the case of $z$-cut PPLN, this scheme works with the proper selection of the azimuthal angle of the crystal. The optical pump pulses propagate in the [100] direction of the PPLN crystal. The optical pulses $\mathbf{E}_{1}$ and $\mathbf{E}_{2}$ are polarized along the [011] and [0-11] directions, respectively. The electric fields of the optical pulses are described as

$$
\begin{aligned}
& \mathbf{E}_{1}(t)=\frac{E_{O}(t)}{\sqrt{2}}(0,1,1), \\
& \mathbf{E}_{2}(t)=\frac{E_{O}(t-\tau)}{\sqrt{2}}(0,-1,1),
\end{aligned}
$$

where $\tau$ is the relative time delay between the optical pump pulses. Each optical pulse generates a linearly polarized multicycle $\mathrm{THz}$ pulse centered at $1.015 \mathrm{THz}$. The crystal class of $\mathrm{LiNbO}_{3}$ is $3 m$, so there are three independent elements of the second order nonlinear susceptibility: $d_{22}, d_{31}$, and $d_{33}$. The generated $\mathrm{THz}$ pulses can then be described as

$$
\begin{aligned}
& \mathbf{E}_{1}^{\mathrm{THz}}(t)=E_{T}(t)\left(0, d_{22}+2 d_{31}, d_{31}+d_{33}\right), \\
& \mathbf{E}_{2}^{\mathrm{THz}}(t)=E_{T}(t-\tau)\left(0, d_{22}-2 d_{31}, d_{31}+d_{33}\right) .
\end{aligned}
$$

Thus the angle between the polarizations of the $\mathrm{THz}$ pulses $\mathbf{E}_{1}^{\mathrm{THz}}$ and $\mathbf{E}_{2}^{\mathrm{THz}}$ is

$$
\theta=\tan ^{-1}\left(\frac{d_{22}+2 d_{31}}{d_{31}+d_{33}}\right)-\tan ^{-1}\left(\frac{d_{22}-2 d_{31}}{d_{31}+d_{33}}\right) .
$$

The experimentally measured angle using a WG $\mathrm{THz}$ polarizer is $6^{\circ}$. The small value of the measured angle indicates that the ellipticity of the superposed $\mathrm{THz}$ waves is weak, and the polarization is almost linear. In the optical regime, the nonlinear susceptibility tensor components are given as $d_{22}=7.4, d_{31}=14$, and $d_{33}=98\left(10^{-9} \mathrm{esu}\right),{ }^{10}$ which corresponds to $28^{\circ}$ for the calculated angle between the nonlinear polarizations. The smaller angle in the $\mathrm{THz}$ regime 


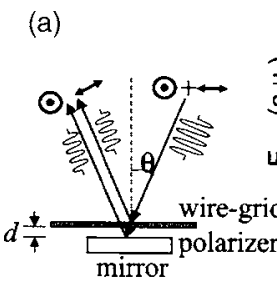

(b)

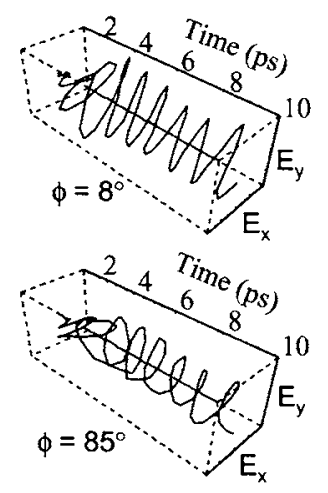

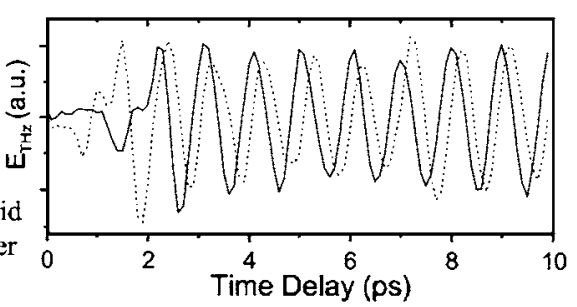

(c)

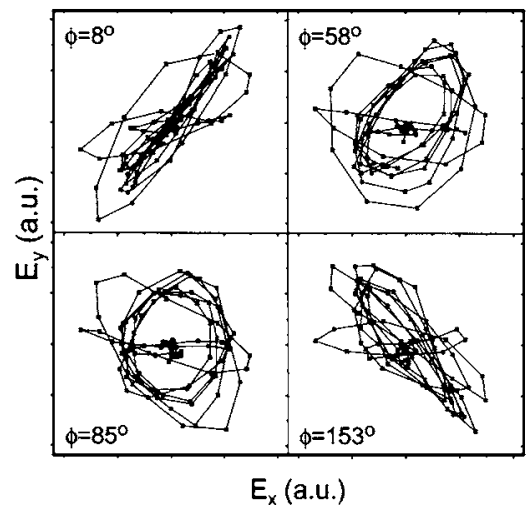

FIG. 3. THz wave plate consisting of WG polarizer and mirror controls $\mathrm{THz}$ ellipticity of narrow-band multicycle THz pulses. (a) Two linearly and perpendicularly polarized $\mathrm{THz}$ pulses $E_{x}$ (dotted line: reflection from the WG polarizer) and $E_{y}$ (solid line: reflection from the mirror) are shown. The phase difference between the THz pulses at $\nu_{\mathrm{THz}}=1.015 \mathrm{THz}$ is $\phi=-78^{\circ}$. (b) 3D plots of $\mathrm{THz}$ E-field vectors for $\phi=8^{\circ}$ and $85^{\circ}$. (c) Polarization trajectories in the $x-y$ plane for $\phi=8^{\circ}, 58^{\circ}, 85^{\circ}$, and $153^{\circ}$.

indicates that the relative nonlinearity in the direction of the optic axis is stronger in the $\mathrm{THz}$ regime than in the optical regime: $d_{33} \gg d_{22}, d_{31}$. Since $\theta \cong 4 d_{31} / d_{33}$, the measurement leads to $d_{31} / d_{33} \cong 0.03$ in the $\mathrm{THz}$ regime. The time delay $(\tau)$ is $0.29 \mathrm{ps}$ corresponding to $106^{\circ}$ of the phase difference between the THz waves at $1.015 \mathrm{THz}$. The superposed THz pulse can be written as

$$
\hat{z}^{\prime} E_{z^{\prime}}^{\mathrm{THz}} \cos \left(k x-\omega_{\mathrm{THz}} t\right)+\hat{y}^{\prime} E_{y^{\prime}}^{\mathrm{THz}} \cos \left(k x-\omega_{\mathrm{THz}} t+\phi\right),
$$

where $E_{y^{\prime}}^{\mathrm{THz}} / E_{z^{\prime}}^{\mathrm{THz}}=0.088$ and $\phi=53^{\circ} . \hat{z}^{\prime}$ and $\hat{y}^{\prime}$ are orthonormal unit vectors and $\hat{z}^{\prime}$ is parallel to the polarization of the THz pulse $\mathbf{E}_{1}^{\mathrm{THz}}$.

Due to the relatively strong nonlinearity along the optic axis, the two-pulse scheme is not a very efficient way to generate circularly polarized $\mathrm{THz}$ waves. An alternative method to control the THz ellipticity is illustrated in Fig. 3(a). Elliptical polarization can be obtained from the reflection of a linearly polarized $\mathrm{THz}$ wave by a combination of a WG polarizer and a mirror. The mirror is placed just behind the polarizer. The mirror and the polarizer reflect two perpendicularly polarized $\mathrm{THz}$ waves. The $\mathrm{THz}$ "wave-plate" (WG polarizer+mirror) controls the ellipticity of the combined $\mathrm{THz}$ waves by adjusting the distance between the mirror and the polarizer and by rotating the polarizer. The $\mathrm{THz}$ wave form shown in Fig. 3(a) is characterized as

$$
\hat{x} E_{x}^{\mathrm{THz}} \cos \left(k z-\omega_{\mathrm{THz}} t\right)+\hat{y} E_{y}^{\mathrm{THz}} \cos \left(k z-\omega_{\mathrm{THz}} t+2 \pi+\phi\right),
$$

where $E_{x}^{\mathrm{THz}} / E_{y}^{\mathrm{THz}}=1.0, \omega_{\mathrm{THz}} / 2 \pi=1.015 \mathrm{THz}$ and $\phi=-78^{\circ}$. The phase resolution at $1.015 \mathrm{THz}$ is $\Delta \phi=0.25^{\circ}$.

Figure 3(b) shows the THz $E$-field vectors of the multicycle narrow-band THz pulses in the $x-y$ plane for $\phi=8^{\circ}$ and $85^{\circ}$. The polarization for $\phi=8^{\circ}$ is almost linear except the first two periods where the two THz pulses only partially overlap. Since the reflection from the WG polarizerlinearly polarized in the $x$ axis - arrives earlier, the trajectory shows the $45^{\circ}$ rotation from the $x$ axis in the first few picoseconds. The early time rotations are also shown in Fig. 3(c): the polarization trajectories in the $x-y$ plane. As the phase increases, the polarization changes continuously: linear $\left(8^{\circ}\right)$ $\rightarrow \operatorname{elliptical}\left(58^{\circ}\right) \rightarrow \operatorname{circular}\left(85^{\circ}\right) \rightarrow \operatorname{elliptical}\left(135^{\circ}\right)$.

In conclusion, we have demonstrated two techniques to generate elliptically polarized $\mathrm{THz}$ pulses. One utilizes optical rectification in nonlinear crystals using two temporally separated optical pump pulses. We control the THz ellipticity adjusting the relative time delay and polarization of the two optical pulses. We generate mixed polarization states of single-cycle $\mathrm{THz}$ pulses using $\mathrm{ZnTe}$, and elliptically polarized multicycle THz pulses in PPLN crystals. The second is essentially a broadband $\mathrm{THz}$ wave-plate using a combination of a wire-grid $\mathrm{THz}$ polarizer and a mirror to transform linearly polarized multicycle $\mathrm{THz}$ pulses into elliptical polarization. The wire-grid $\mathrm{THz}$ wave plate is applicable to the entire $\mathrm{THz}$ spectral range. In general, the wave-plate method should be more practical than the two-pulse technique because the set-up is simpler and the polarization control is more flexible. We expect that $\mathrm{THz}$ ellipticity control techniques may be useful for coherent control of carrier dynamics in semiconductor nanostructures and of molecular dynamics. For example, single-cycle $\mathrm{THz}$ pulses of spiral polarization might be useful for exploring quantum chaos of the transiently kicked quantum rotor. ${ }^{11}$ This problem has been rigorously studied theoretically, but only a few experimental studies have been done in atomic systems. ${ }^{12,13} \mathrm{~A}$ spirally polarized single-cycle $\mathrm{THz}$ pulse could be used as a transient rotational impact on gas phase heteropolar molecules, which are nearly ideal quantum rotors. THz timedomain spectroscopy, which can measure not only the amplitude of the rotational motion but also the phase, would be an excellent experimental scheme to investigate the quantum wavepacket dynamics.

This work was supported by NSF CAREER Award No. 0449426 and by DARPA under Grant No. FA9550-04-10465 .

${ }^{1}$ S. Hughes and D. S. Citrin, J. Opt. Soc. Am. B 17, 128 (2000).

${ }^{2}$ J. L. McHale, Molecular Spectroscopy (Prentice Hall, Englewood Cliffs, NJ, 1999), pp. 209-244.

${ }^{3}$ J. Xu, J. Galan, G. Ramian, P. Savvidis, A. Scopatz, R. R. Birge, S. J. Allen, and K. Plaxco, Proc. SPIE 5268, 19 (2004).

${ }^{4}$ J. I. Dadap, J. Shan, and T. F. Heinz, Pacific Rim CLEO Technical Digest 2000, pp. 55-56.

${ }^{5}$ J. Van Rudd, J. L. Jonson, and D. M. Mittleman, J. Opt. Soc. Am. B, 18, 1524 (2001).

${ }^{6}$ Y.-S. Lee, T. Meade, V. Perlin, H. Winful, T. B. Norris, and A. Galvanauskas, Appl. Phys. Lett. 76, 2505 (2000).

${ }^{7}$ Y.-S. Lee, T. Meade, M. DeCamp, T. B. Norris, and A. Galvanauskas, Appl. Phys. Lett. 77, 1244 (2000).

${ }^{8}$ Y.-S. Lee, T. Meade, T. B. Norris, and A. Galvanauskas, Appl. Phys. Lett. 78, 3583 (2001).

${ }^{9}$ Q. Wu and X.-C. Zhang, Appl. Phys. Lett. 68, 1604-1606(1996).

${ }^{10}$ R. W. Boyd, Nonlinear Optics (Academic, New York, 1992), p. 44.

${ }^{11}$ R. Alicki, A. Kakowec, and W. Miklaszewski, Phys. Rev. Lett. 77, 838 (1996).

${ }^{12}$ H. Ammann, R. Gray, I. Shvarchuck, and N. Chrestensen, Phys. Rev. Lett. 80, 4111 (1998).

${ }^{13}$ B. G. Klappauf, W. H. Oskay, D. A. Steck, and M. G. Raizen, Phys. Rev. Lett. 81, 1203 (1998). 